\title{
Simplifying the diagnosis of hemophilia
}

\author{
Laura Villarreal-Martínez and Cynthia A. Salinas-Silva \\ Hematology Service, Dr. José Eleuterio González University Hospital, Universidad Autónoma de Nuevo León, Monterrey, N.L., Mexico
}

Hemophilia is a hereditary disorder linked to the $\mathrm{X}$ chromosome, where there are mutations in the genes of coagulation Factors VIII and IX, causing functional or quantitative deficiencies giving rise to hemophilia $A$ (Factor VIII deficiency) and hemophilia B (Factor IX deficiency). These mutations cause an alteration in secondary hemostasis ${ }^{1}$. Hemophilia $A$ has a worldwide incidence of 1 in 5,000 male births, and hemophilia B has an incidence of 1 in 30,000 male births, mainly affecting men, women are the carriers and transmitters of this disease. According to the report of the 2016 Annual Global Survey, there are 5,693 people with hemophilia in Mexico ${ }^{2} .70 \%$ of patients with hemophilia have family history with this disease, and the remaining $30 \%$ have de novo mutations ${ }^{3}$. Hemophilia is classified according to the percentage of the functional level of the affected factor; the normal reference values are 50$150 \%^{4}$ (Table 1).

The symptoms of hemophilia are directly related to its severity. The lower the percentage of activity of the factor involved, the greater the number of bleeding episodes. In severe hemophilia, spontaneous and traumatic hemorrhages may occur, being the joints the most affected. In general, in severe hemophilia, patients are diagnosed in the early stages of life. Bleeding may occur in soft tissues of the head caused by the passage of the newborn through the birth canal, muscle bruising may show after the application of Vitamin $\mathrm{K}$ prophylaxis or vaccines, and hemarthrosis when they start crawling.
In moderate hemophilia, spontaneous hemorrhages are less frequent. However, prolonged hemorrhages may develop, caused by surgical interventions, dental procedures, or secondary to minor trauma. In mild hemophilia, the occurrence of spontaneous hemorrhages is rare and only happens due to sustained major trauma or during surgery. Due to the variations in the clinical presentation, the diagnosis may be delayed, particularly in patients with moderate and mild forms, which may not be diagnosed until adulthood.

The characteristic manifestation is the tendency to hemorrhage mainly in the large joints, in muscles, in the mucous membranes of the mouth, the gums, the nose, and the genitourinary tract. Hemorrhages may also occur in locations that endanger life, such as in the central nervous system, in the neck/throat, and in the gastrointestinal system. The incidence of bleeding is shown in Table $2^{5}$.

The diagnosis of hemophilia begins with clinical suspicion (Fig. 1). When we consider that a patient may have hemophilia or another bleeding disorder, it is essential to carry out initial laboratory studies. The following basic studies should be requested: a complete blood count, activated partial thromboplastin time (aPTT), and prothrombin time (PT). In hemophilia, the platelet count is normal, the PT is normal, and the aPTT is prolonged, usually in more than two standard deviations. Therefore, the mixing tests should be performed, mixing the plasma of the patient with normal plasma at a 1:1 ratio. If the aPTT is "corrected," it shows

\section{Correspondence:}

Laura Villarreal-Martínez

E-mail: dr_lauravillarreal@ hotmail.com
Date of reception: 07-08-2018

Date of acceptance: 04-09-2018

DOI: 10.24875/RMU.M18000024
Available online: $30-11-2018$

Medicina Universitaria. 2018;20(3):133-135 www.medicinauniversitaria.org CC BY-NC-ND license (http://creativecommons.org/licenses/by-nc-nd/4.0/). 


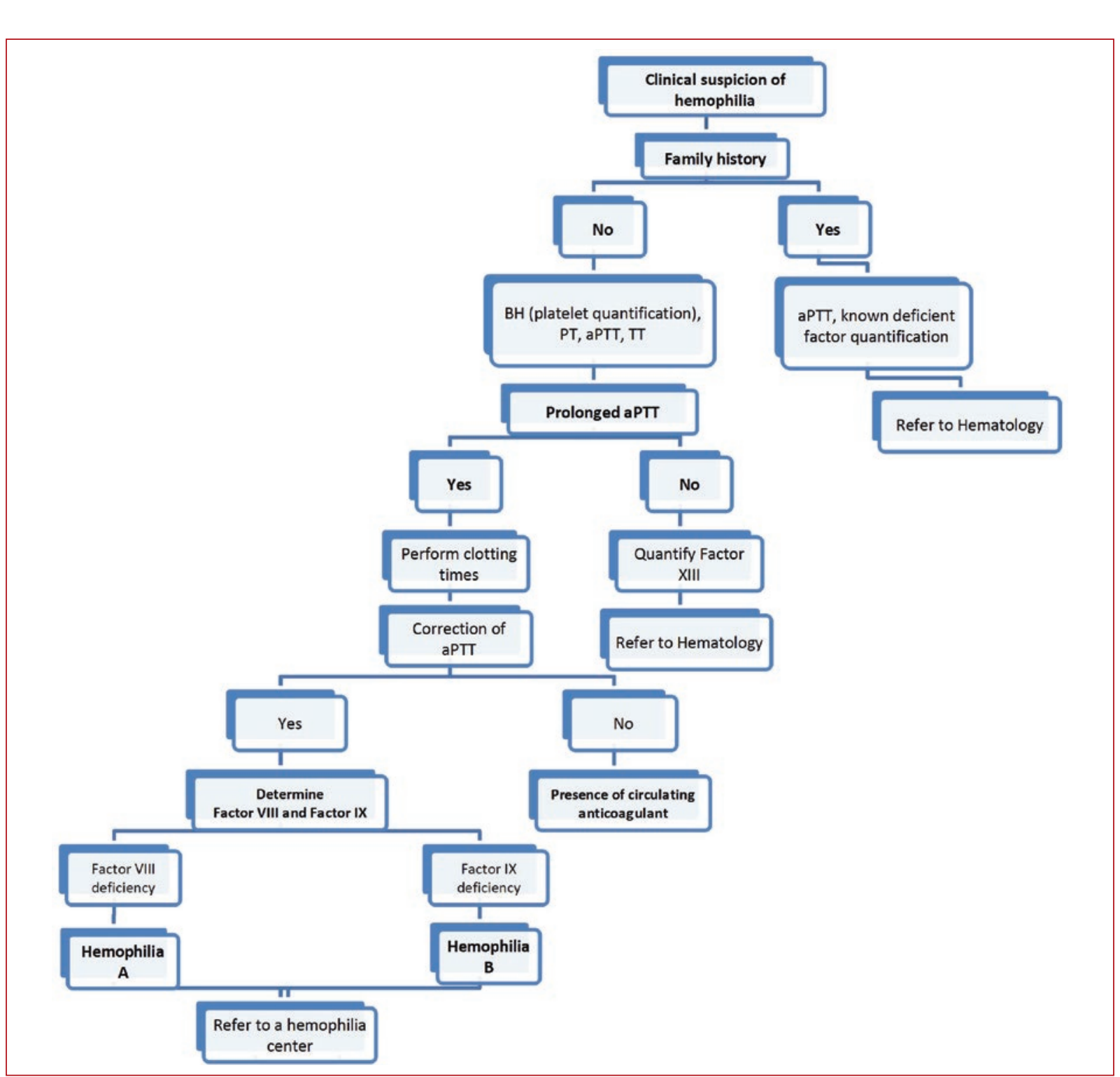

Figure 1. Clinical diagnostic approach of hemophilia.

a deficiency of a coagulation factor. It is necessary to quantify the activity of the VIII or IX coagulant clotting factors, using chromogenic or coagulometric methods, which allow us to pinpoint low levels to confirm the diagnosis, once the deficient factor has been identified, the patient should be referred to a specific hemophilia center ${ }^{5}$.

In males with a known history of hemophilia, children of possible carriers, and/or who present hemorrhagic manifestations, the aPTT should be measured directly, and the known deficient factor quantified at birth or the moment of its first hemorrhagic manifestation, to confirm the diagnosis. It is recommended to refer patients with: prolonged aPTT, hemorrhage with severity more significant than expected, and newborns with a hereditary family history of hemophilia or some hemorrhagic manifestation, to a second or third level of care.

The main goal of the treatment of hemophilia is to prevent and treat hemorrhages promptly to preserve normal function of the musculoskeletal system. This is achieved through the intravenous administration of the deficient factor. However, its costs and the need for frequent intravenous infusions increase the difficulty in carrying it out. There are two types of concentrated factors for treatment: the plasma-derived factor and the recombinant factor. 
Table 1. Classification of the severity of hemophilia.

\begin{tabular}{|l|c|c|}
\hline Classification & $\begin{array}{c}\text { Activity level of the } \\
\text { coagulation factor (\%) }\end{array}$ & $\begin{array}{c}\text { Number of IU/ml of } \\
\text { total blood }\end{array}$ \\
\hline Severe & $<1$ & $<0.01 \mathrm{UI} / \mathrm{ml}$ \\
\hline Moderate & $1-5$ & $0.01-0.05 \mathrm{UI} / \mathrm{ml}$ \\
\hline Mild & $5-40$ & $0.05-0.40 \mathrm{UI} / \mathrm{ml}$ \\
\hline
\end{tabular}

Table 2. Sites and incidence of hemorrhages in hemophilia.

\begin{tabular}{|l|c|}
\hline Location of hemorrhage & Incidence (\%) \\
\hline Hemarthrosis & $70-80$ \\
Knee & 45 \\
Elbow & 30 \\
Ankle & 15 \\
Doll & 3 \\
Hip & 2 \\
\hline Muscles & $10-20$ \\
\hline Other hemorrhages & $5-10$ \\
\hline Central nervous system & $<5$ \\
\hline
\end{tabular}

The different modalities of treatments are primary prophylaxis, secondary prophylaxis, on demand, and domiciliary treatment. Treatment should be individualized according to each patient needs prophylaxis regimen is adapted according to the weight of the patient. The Malmö protocol can be used, where 25-40 IU/kg is given 3 times a week in hemophilia A (Factor VIII) and twice a week in hemophilia B (Factor IX). The Utrecht protocol can also be used, giving $15-30 \mathrm{IU} / \mathrm{kg}$ 3 times a week in hemophilia $A$ and twice in hemophilia $B$. In countries with little access to factor concentrates, administering 10-15 IU/kg 3 times a week can be an effective option ${ }^{6}$.

In acute hemorrhages, the immediate application of factor replacement therapy is required in the first 2 hours after the clinical manifestation, along with rest, ice application, compression, and elevation. Adjuvant therapies such as desmopressin or tranexamic acid can be used to control bleeding if concentrates of the required factor are not available. For analgesia, the use of drugs that affect platelet function should be avoided, such as aspirin and the group of nonsteroidal anti-inflammatories, except for COX-24,5 inhibitors. In case of pain, paracetamol or an opioid derivative can be used.

Patients can practice low-impact activities such as swimming, walking, and cycling with adequate protection, and avoiding high-impact activities that could cause trauma. Orthopedic and physical medicine and rehabilitation physicians must be part of the multidisciplinary team.

Complications of hemophilia are permanent sequelae caused by hemorrhages in the musculoskeletal and central nervous systems, viral infections that can be transmitted through transfused blood products, and the development of inhibitors targeting the administered coagulation factors. Inhibitor development, where there is a formation of antibodies against the exogenous factor administered during treatment deactivating the coagulant activity, is the most severe complication in hemophilia occurring in $30-40 \%$ of patients ${ }^{7}$, being more common in hemophilia A. Presence of inhibitors should be suspected in patients in whom favorable results are not obtained with the standard treatment.

In conclusion, it is very important to know the forms of presentation, manifestations, and diagnostic strategies of the patient with hemophilia and refer the patient promptly to a specialized center to reduce their morbidity mortality as much as possible.

\section{References}

1. Bolton-Maggs PH, Pasi KJ. Haemophilias A and B. Lancet. 2003;361:1801-9.

2. World Federation of Hemophilia. Report on the Annual Global Survey 2016. Canadá: World Federation of Hemophilia; 2017.

3. Osorio-Guzmán M. Percepción de la calidad de vida relacionada con la salud y la depresión en pacientes con hemofilia. Rev Méd Inst Mex Seguro Soc 2017;55:4.

4. Srivastava A, Brewer AK, Mauser-Bunschoten EP, et al. Guidelines for the management of hemophilia. Haemophilia. 2013;19:e1-47.

5. Diagnóstico y Tratamiento de Hemofilio Pediátrica. En Catálogo Maestro de Guías de Prática Clínica: IMSS-141-08. México, D.F.: Centro Nacional de Excelencia Tecnológico en Salud; 2012.

6. Castaman G, Linari S. Prophylactic versus on-demand treatments for hemophilia: advantages and drawbacks. Expert Rev Hematol. 2018;11:567-76.

7. Carcao M, Shapiro A, Staber JM, et al. Recombinant factor VIII fc fusion protein for immune tolerance induction in patients with severe haemophilia A with inhibitors-A retrospective analysis. Haemophilia. 2018;24:245-52. 